\title{
Association between perilipin gene polymorphisms and body weight traits in Jinmao Hua chickens
}

\author{
Tingting $\mathrm{Li}^{1{ }^{1,}}$, Jiaojiao Ling ${ }^{1, *}$, Lian Duan ${ }^{2}$, Qian Xue ${ }^{1}$, and Jinyu Wang ${ }^{1}$ \\ ${ }^{1}$ College of Animal Science and Technology, Yangzhou University, Yangzhou, Jiangsu, China \\ ${ }^{2}$ Jiangsu Institute of Poultry Science, Chinese Academy of Agricultural Science, Yangzhou, China \\ *These authors contributed equally to this work.
}

Correspondence to: Jinyu Wang (jywang@yzu.edu.cn)

Received: 21 March 2017 - Revised: 18 August 2017 - Accepted: 24 August 2017 - Published: 27 September 2017

\begin{abstract}
The perilipin gene ( $P L I N)$ plays a crucial role in lipid metabolism and fat deposition. In order to reveal the genetic effects of PLIN polymorphisms on body weight (BW) traits in chickens, PLIN gene polymorphisms in 322 Jinmao Hua chickens were detected by PCR-SSCP and DNA sequencing methods. For PLIN primer pair 1, five genotypes (AA, AB, BB, JJ and JL) were detected in the Jinmao Hua chicken population and three mutations (g.1889C > T, g.1904T > C and g.1922C > T) were revealed by gene sequencing. For PLIN primer pair 2, three genotypes (CC, CD and DD) were detected in the same population and two mutations (g.2014A > G and g.2020C $>$ T) were revealed by gene sequencing. Least squares analysis showed that individuals with the $\mathrm{JJ}$ and CD genotypes performed better than the other Jinmao Hua chicken genotypes. Based on the five SNPs, the frequency distributions of the eight haplotypes were estimated with PHASE2.1 software. C-T-C-G-T was the major haplotype with a frequency of $58.6957 \%$, while the frequency of C-C-C-A-C was less than $1 \%$. Fourteen diplotypes were obtained from the eight haplotypes. $\mathrm{H} 1 \mathrm{H} 1$ was the dominant diplotype with a frequency of $47.205 \%$. Least squares analysis indicated that $\mathrm{BW}$ with the $\mathrm{H} 3 \mathrm{H} 3$ diplotype was the lowest, while the $\mathrm{H} 2 \mathrm{H} 2$ diplotype was the highest, suggesting that selecting for the $\mathrm{H} 3 \mathrm{H} 3$ diplotype improved the $\mathrm{BW}$ traits of Jinmao Hua chickens. The findings of this study should be useful to expand the theoretical basis of the role the PLIN in poultry molecular breeding of poultry.
\end{abstract}

\section{Introduction}

Perilipin, a PAT (perilipin/adipophilin/tail-interacting protein of $47 \mathrm{kDa}$ ) family protein and encoded by the PLIN gene, is a lipid droplet-associated phosphor-protein that functions as a key regulator during lipid metabolism and fat deposition that plays an important role in the formation and maintenance of lipid droplet (Londos et al., 2005; Liu and Xu, 2006). Unphosphorylated perilipin molecules coat the surface of lipid droplets in adipocytes to form a barrier that prevents lipase from accessing triacylglycerol, thereby inhibiting lipolysis (Wolins et al., 2005; Xu et al., 2006). Upon activation of protein kinase A, hormone-sensitive lipase (HSL) and perilipin are phosphorylated. Phosphorylation of perilipin is essential for the translocation of HSL from the cytoplasm to lipid droplets, thus perilipin phosphorylation is an impor- tant molecular switch for the activation of lipolytic reactions (Londos et al., 1999; Xu et al., 2004).

Studies indicate that the PLIN gene affects body weight (BW) and fat deposition in animals (Londos et al., 2005; Bickel et al., 2009). Mutations to PLIN have been associated with carcass traits and adiposity in humans (Lü et al., 2015), pigs (Gandolfi et al., 2011; Gol et al., 2015), cattle (Fan et al., 2010), sheep (Gao et al., 2012), and ducks (Zhang et al., 2013). To date, only three reports have investigated the association of PLIN polymorphisms with a limited number of production traits in chickens. In the first report, three singlenucleotide polymorphisms (SNPs) of PLIN (g.2272C > T, g.2319C > T, and g.2476G $>$ A) were significantly associated with living body weight, carcass weight, and leg muscle weight, and strongly correlated to abdominal fat weight and abdominal fat percentage (Zhou et al., 2014). In the second 
Table 1. Primer sequences of PCR amplification of chicken PLIN gene.

\begin{tabular}{llrr}
\hline Primer & Sequences $\left(5^{\prime}-3^{\prime}\right)$ & Length of products $(\mathrm{bp})$ & Annealing temp. $\left({ }^{\circ} \mathrm{C}\right)$ \\
\hline PLIN Primer 1 & F: CTCTGTGCTGTTCTGCCTTTA & 242 & 58.9 \\
& R: ATCCAGACGACCAGTTCCTG & & \\
PLIN Primer 2 & F: AAAGCCAAAGGGCAGGAA & 114 & 56.3 \\
& R: TGCAGAGGGAACAGAAACC & & \\
\hline
\end{tabular}

study, a SNP located at rs 315831750 on chromosome 10 was found to be associated with the percentages of breast muscle, leg muscle, and abdominal fat (Zhang et al., 2015). Certainly, not all genetic variations exert distinct effects on carcass and adipose traits, as concluded by the third study, which found no significant correlation of a single mutation (g.2224G $>\mathrm{T}$ ) of the PLIN gene with carcass and abdominal traits (Lei et al., 2011).

Hence, most studies on PLIN gene polymorphisms in chickens have focused on carcass and abdominal traits, while few have investigated the effect on BW traits. Therefore, the aim of the present study was to further investigate the contribution of the PLIN gene to a wider range of performance in chickens, especially to confirm whether PLIN polymorphisms exert a significant effect on BW at different ages.

\section{Materials and methods}

\subsection{Ethics statement}

The study protocol was approved by the Animal Care Committee of the Department of Animal Science and Technology of Yangzhou University and conducted in accordance with the guidelines of the Animal Use Committee of the Chinese Ministry of Agriculture. All efforts were made to minimize animal suffering.

\subsection{Population and samples collection}

A total of 322 female Jinmao Hua chickens were selected randomly from the same feeding batch at Jiangsu Sanditi Animal Husbandry Co., Ltd. All birds were hatched on the same day and reared on the ground under the same nutritional and environmental conditions. Six growth traits, the BW of chickens at day 0 (BW0) and weeks 2 (BW2), 4 (BW4), 6 (BW6), 8 (BW8) and 10 (BW10), were recorded. Genomic DNA was extracted by the phenol-chloroform extraction method, dissolved in Tris-ethylenediaminetetraacetic acid (EDTA) buffer, then quantified by spectrophotometry and then stored at $-20^{\circ} \mathrm{C}$ until analyzed.

\subsection{Primer design and variability analysis}

According to the gallinaceous PLIN gene sequences (GenBank Accession no. NC_006097.3), Primer 5.0 software was used to design two pairs of primers to amplify fragments

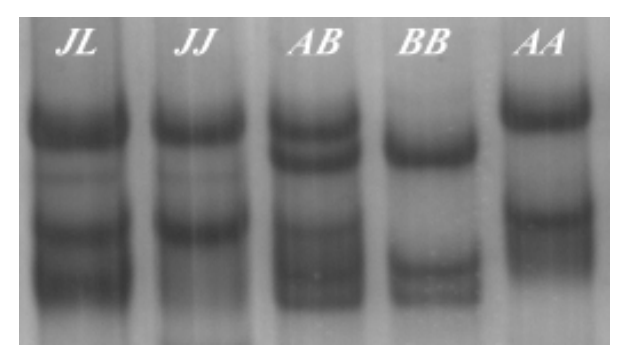

Figure 1. Polyacrylamide gel electrophoresis results of SSCP analysis of PCR products in Jinmao Hua chickens.

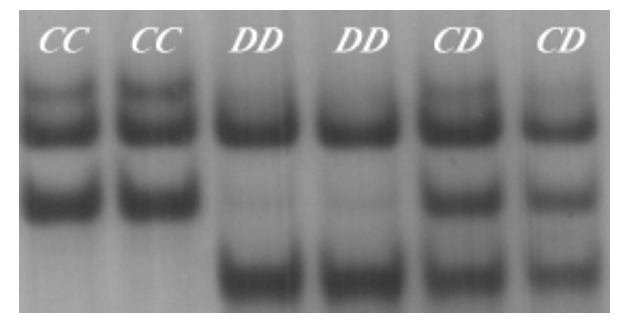

Figure 2. SSCP analysis of PCR amplification using primer 2 in Jinmao Hua chickens.

that encompassed exon 5 and a partial intron of the PLIN gene (Table 1). Primers were synthesized by Sangon Biotech (Shanghai) Co., Ltd. Polymerase chain reaction (PCR) was performed in a $20 \mu \mathrm{L}$ reaction volume containing $1 \mu \mathrm{L}$ of genomic DNA, $0.8 \mu \mathrm{L}$ of each primer, $7.4 \mu \mathrm{L}$ of $\mathrm{dH}_{2} \mathrm{O}$, and $10 \mu \mathrm{L}$ of $2 \times$ Taq Master Mix for polyacrylamide gel electrophoresis (Vazyme Biotech Co., Ltd., Nanjing, China). The PCR thermal profile consisted of pre-denaturation at $95^{\circ} \mathrm{C}$ for $5 \mathrm{~min}$, followed by 30 cycles of denaturation at $95^{\circ} \mathrm{C}$ for $30 \mathrm{~s}$, annealing at $X^{\circ} \mathrm{C}$ for $30 \mathrm{~s}$ and elongation at $72^{\circ} \mathrm{C}$ for $30 \mathrm{~s}$ and final extension at $72^{\circ} \mathrm{C}$ for $10 \mathrm{~min}(X$ is the annealing temperature specific for the primer pairs; see Table 1).

For single-strand conformation polymorphism (SSCP) analysis, $2 \mu \mathrm{L}$ of each amplification product was mixed with $7 \mu \mathrm{L}$ of denaturing buffer ( $98 \%$ formamide, $0.025 \%$ bromophenol blue, $0.025 \%$ xylene cyanol FF, $10 \mathrm{mmol} /$ LEDTA (at $\mathrm{pH} \mathrm{8.0)}$ and $2 \%$ glycerol), heated for $10 \mathrm{~min}$ at $98^{\circ} \mathrm{C}$ and then cooled on ice for $10 \mathrm{~min}$. Denatured PCR products were subjected to $10 \%$ non-denaturing polyacrylamide (29: 1) gel electrophoresis at $250 \mathrm{~V}$ for the first $5 \mathrm{~min}$ and then $120 \mathrm{~V} \mathrm{~cm}^{-1}$ for 10 to $12 \mathrm{~h}$. SSCP patterns on the gels 


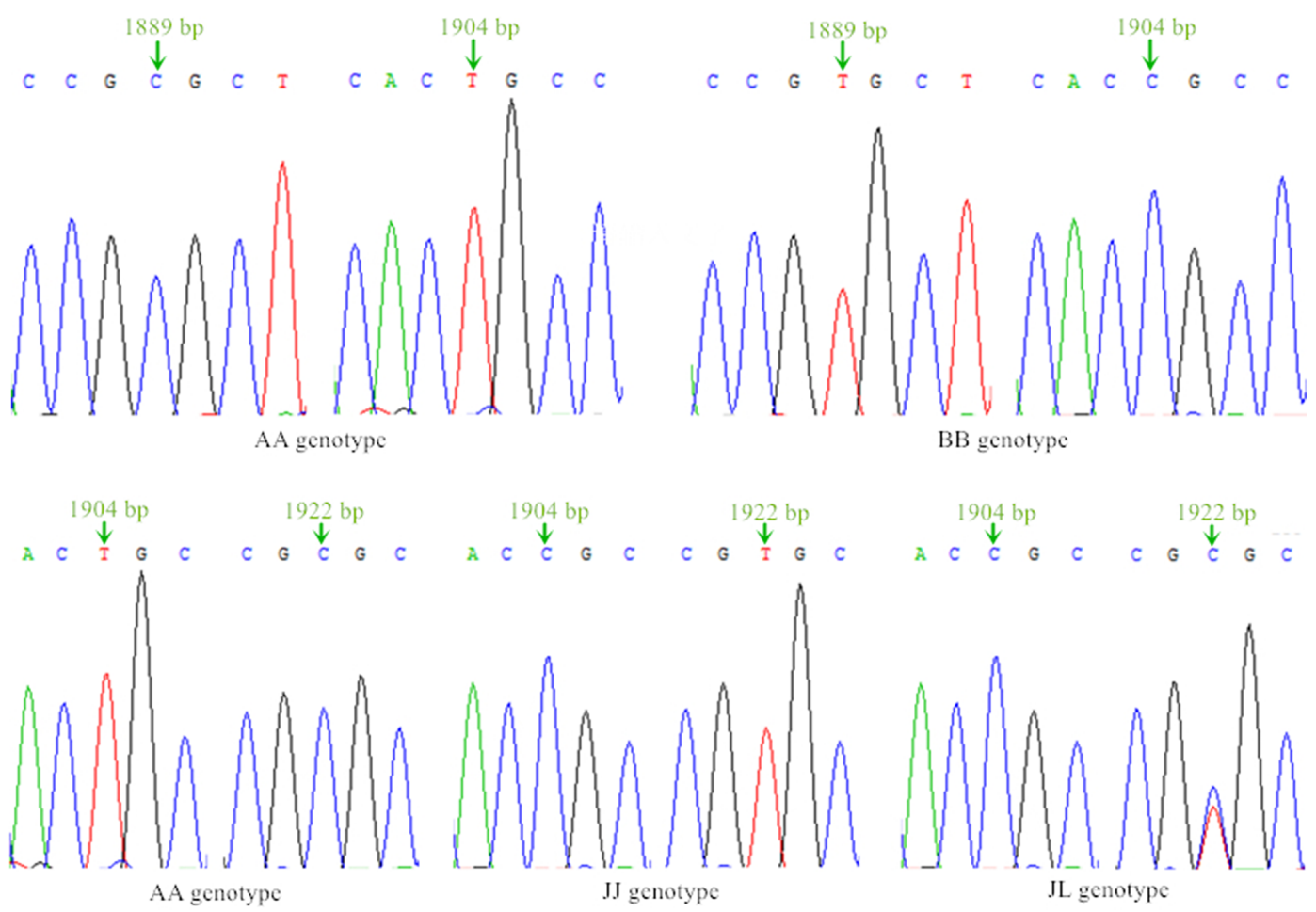

Figure 3. Sequence alignment of AA, BB, JJ and JL genotypes of primer 1.

were visualized by silver staining. For each genotype, Sangon Biotech sequenced the PCR products of four samples.

\subsection{Statistical analysis}

PHASE2.1 software (http://stephenslab.uchicago.edu/phase/ download.html) was used to analyze the types and frequency of haplotypes. The association between genotypes and growth traits were analyzed using the general liner model (GLM) procedures of SPSS ver. 19.0 software (SPSS, Inc., Chicago, IL, USA). The following statistical model was used:

$Y=\mu+G_{i}+e$,

where $Y$ is the phenotypic value of traits, $\mu$ the population mean, $G_{i}$ fixed effects of genotype or diplotype, and $e$ random residual error. Multiple comparisons were performed with least squares means.

\section{Results}

\subsection{SSCP and sequence analysis}

SSCP analysis revealed that the products of the two primer pairs displayed polymorphisms in the PLIN gene. Five genotypes were detected in Jinmao Hua chickens with primer pair 1 (AA, AB, BB, JJ and JL; Fig. 1) and three with primer pair 2 (CC, CD and DD; Fig. 2).
The PCR products of different genotypes were cloned and sequenced. Sequencing revealed two nucleotide mutations (g.1889C $>\mathrm{T}$ and g.1904T $>$ C) in the AA genotype, as compared with BB genotypes, and two nucleotide mutations (g.1904T > C and g.1922C > T) between the JJ and $\mathrm{JL}$ genotypes. Individuals with the JJ genotype had one nucleotide mutation (g.1922C > T), as compared with the JL genotype (Fig. 3). Sequencing analysis results of the different genotypes of primer pair 2 are depicted in Fig. 4. Two mutations were detected by sequencing (g.2014A $>\mathrm{G}$ and g. $2020 \mathrm{C}>\mathrm{T}$ ). None of these mutations resulted in an amino acid change.

\subsection{Association of genotypes with BW traits}

One-way analysis of variance was used to analyze the effects of different genotypes. The use of primer pair 1 revealed that the polymorphism was significantly associated with growth traits in of Jinmao Hua chickens on BW0, BW6 and BW8 (Table 2). Genotype JJ had a significantly higher hatch weight than that of the BB and JL genotypes $(P<0.05)$. The JJ genotype was predominant, as compared with the JL genotype $(P<0.05)$, at 6 weeks of age. At BW8, chickens with the JJ genotype were significantly heavier than the JL chickens. There was no distinct difference in the other traits among the five genotypes $(P>0.05)$.

The results of multiple comparison analysis of growth traits between the genotypes for primer pair 2 among all 
Table 2. Parameter estimates of different genotypes on body weight traits for primer 1 (means \pm SD).

\begin{tabular}{lrrrrr}
\hline Traits & \multicolumn{5}{c}{ Genotype } \\
\cline { 2 - 6 } & AA $(N=209)$ & $\mathrm{BB}(N=9)$ & $\mathrm{AB}(N=33)$ & $\mathrm{JJ}(N=41)$ & $\mathrm{JL}(N=30)$ \\
\hline BW0 & $25.10 \pm 2.01^{\mathrm{ab}}$ & $23.07 \pm 2.54^{\mathrm{b}}$ & $24.73 \pm 2.39^{\mathrm{ab}}$ & $25.60 \pm 2.36^{\mathrm{a}}$ & $24.49 \pm 2.08^{\mathrm{b}}$ \\
BW2 & $108.21 \pm 16.67$ & $105.68 \pm 14.86$ & $111.00 \pm 15.68$ & $112.04 \pm 17.25$ & $108.17 \pm 20.67$ \\
BW4 & $230.03 \pm 39.86$ & $232.38 \pm 43.84$ & $226.88 \pm 36.87$ & $235.21 \pm 39.44$ & $219.37 \pm 42.57$ \\
BW6 & $400.54 \pm 64.02^{\mathrm{ab}}$ & $408.32 \pm 70.04^{\mathrm{ab}}$ & $392.88 \pm 60.31^{\mathrm{ab}}$ & $415.07 \pm 58.14^{\mathrm{a}}$ & $379.24 \pm 62.97^{\mathrm{b}}$ \\
BW8 & $635.18 \pm 87.08^{\mathrm{ab}}$ & $633.68 \pm 98.51^{\mathrm{ab}}$ & $610.97 \pm 96.45^{\mathrm{b}}$ & $653.83 \pm 83.60^{\mathrm{a}}$ & $608.19 \pm 96.16^{\mathrm{b}}$ \\
BW10 & $849.93 \pm 111.91$ & $849.81 \pm 106.61$ & $824.93 \pm 123.39$ & $864.07 \pm 97.45$ & $827.52 \pm 108.84$ \\
\hline
\end{tabular}

Note: different letters in the rows indicate significantly different mean value at $P<0.05$ and the same letters indicate no significant difference $(P>0.05)$.
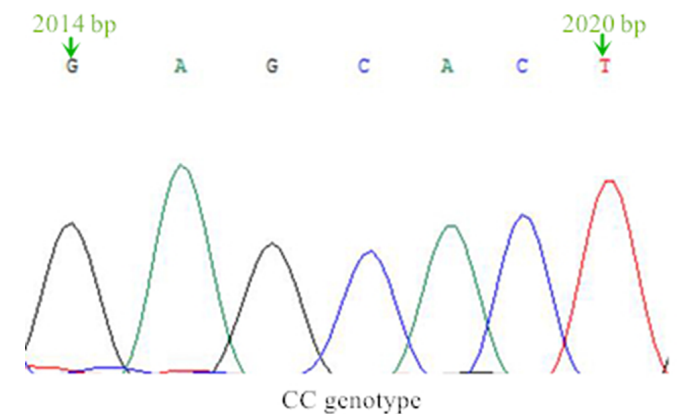

Figure 4. Sequence alignment of CC and DD genotypes of primer 2.
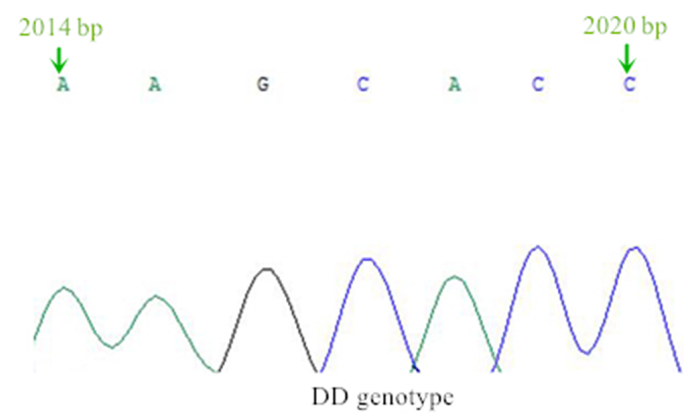

322 female Jinmao Hua chickens are presented in Table 3. Significant differences among the three genotypes were found at BW8 and BW10 $(P<0.05)$. Chickens of genotype $\mathrm{CD}$ had the highest BW and those with the DD genotype had the lowest. No difference was detected among three genotypes at BW0, BW2, BW4 and BW6.

\subsection{Construction of haplotype and association analysis}

The parameters of the haplotypes based on the five SNPs are shown in Table 4. A total of eight haplotypes were detected, with C-T-C-G-T as the main haplotype, accounting for $58.6957 \%$ of the observations. Based on the eight haplotypes, 14 diplotypes in Jinmao Hua chickens were obtained. To ensure that the analysis was accurate, haplotypes and diplotypes with a frequency of less than $1 \%$ were excluded from further association analysis. The frequencies of haplotypes and displotypes are listed in Tables 4 and 5 .

The least squares mean multiple comparisons of diplotypes are displayed in Table 6. Diplotypes were found to be strongly significantly associated with BW6 $(P=0.027)$ and BW8 $(P=0.013)$. For BW0 and BW4, there was no significant association although a trend was observed $(P=0.093$ and 0.067 , respectively). The BW of chickens with the $\mathrm{H} 2 \mathrm{H} 2$ was greater than the other eight diplotypes at each week. Meanwhile, the BW0, BW2, BW4, BW6, BW8 and BW10 values of chickens with the $\mathrm{H} 3 \mathrm{H} 3$ diplotype were the lowest.

\section{Discussion}

\subsection{Polymorphisms of the PLIN gene}

According to the dbSNP database of the National Center for Biotechnology Information, the human PLIN gene has 555 SNPs, including some that may be closely associated to obesity, lipid metabolism, etc. In animals, the PLIN gene also shows abundant polymorphism. Fan et al. (2010) found two SNPs located in the region of exon 3 and 4 in Qinchuan cattle. Gao et al. (2012) detected mutations in exon 3, 4, 5, 6 and 8 in sheep. Fan et al. (2011) found one SNP in exon 2 and in intron 1 of the PLIN gene in Peking as well as Cherry Valley ducks. Vykoukalová et al. (2009) used PCR-restriction fragment length polymorphism to scan the overlapping PCR fragments covering the porcine PLIN gene from exon 1 to 9 and detected eight SNPs in the coding sequence of the porcine PLIN gene. Zhang et al. (2015) designed primers that covered all eight exons to detect the PLIN polymorphisms in six broiler populations, and discovered only one SNP (rs313726543) in exon 1. Additionally, the study on Luqin chickens revealed three SNPs, two in exon 6 and one in intron 6 (Zhou et al., 2014).

We scanned the SNPs in exon 5 and partial intron 5 regions of the PLIN gene of Jinmao Hua chickens. The products amplified by PLIN primer pair 1 and 2 displayed polymorphism and five SNPs were identified by sequencing of the two PCR fragments, including three (g.1889C $>$ T, g.1904T $>$ C and 
Table 3. Parameter estimates of different genotypes on growth traits for primer 2 (means $\pm \mathrm{SD}$ ).

\begin{tabular}{lrrrrrr}
\hline Genotype & \multicolumn{7}{c}{ Traits } \\
\cline { 2 - 7 } & BW0 & BW2 & BW4 & BW6 & BW8 & BW10 \\
\hline CC (239) & $24.99 \pm 2.18$ & $109.48 \pm 17.32$ & $230.20 \pm 9.54$ & $399.65 \pm 63.15$ & $633.12 \pm 86.11^{\text {ab }}$ & $845.21 \pm 106.37^{\text {ab }}$ \\
CD (64) & $25.24 \pm 2.12$ & $107.94 \pm 16.60$ & $231.00 \pm 38.42$ & $407.27 \pm 57.14$ & $641.57 \pm 84.33^{\mathrm{a}}$ & $868.29 \pm 99.99^{\mathrm{a}}$ \\
DD (19) & $24.53 \pm 1.70$ & $105.02 \pm 15.00$ & $214.64 \pm 42.03$ & $377.18 \pm 64.46$ & $594.45 \pm 94.94^{\mathrm{b}}$ & $799.16 \pm 129.68^{\mathrm{b}}$ \\
\hline
\end{tabular}

Note: different letters in the rows indicate significantly different mean value at $P<0.05$ and the same letters indicate no significant difference $(P>0.05)$.

Table 4. Haplotypes inferred on the five single-nucleotide polymorphisms.

\begin{tabular}{llllllr}
\hline Haplotype & g. 1889C $>$ T & g. 1904T $>$ C & g.1922C $>$ T & g. 2014A $>$ G & g.2020C $>$ T & Frequency (\%) \\
\hline H1 & C & T & C & G & T & 58.6957 \\
H2 & C & C & T & G & T & 15.5280 \\
H3 & C & T & C & A & C & 11.3354 \\
H4 & T & C & C & G & T & 5.4348 \\
H5 & C & C & C & G & T & 4.5031 \\
H6 & T & C & C & A & C & 2.4845 \\
H7 & C & C & T & A & C & 1.8634 \\
H8 & C & C & C & A & C & 0.1553 \\
\hline
\end{tabular}

Table 5. Diplotypes and frequencies of five SNPs.

\begin{tabular}{lrlr}
\hline Diplotype & Frequency $(\%)$ & Diplotype & Frequency $(\%)$ \\
\hline H1H1 & 47.205 & H3H6 & 2.795 \\
H1H3 & 15.528 & H4H4 & 1.8634 \\
H1H4 & 6.8323 & H1H6 & 0.6211 \\
H2H2 & 10.2484 & H4H6 & 0.3106 \\
H2H5 & 8.0745 & H5H7 & 0.9317 \\
H2H7 & 2.4845 & H6H6 & 0.6211 \\
H3H3 & 2.1739 & H7H8 & 0.3106 \\
\hline
\end{tabular}

g.1922C > T) located in exon 5 that formed five genotypes (AA, AB, BB, JJ and JL), while others (g.2014A $>$ G and g.2020C $>$ T) located in intron 5 formed three genotypes (CC, CD and DD). These results combined with of those previous reports (Fan et al., 2010, 2011; Gao et al., 2012; Vykoukalová et al., 2009) indicated that the PLIN gene had an abundance of polymorphism in different animal breeds that conform to the comprehensive functions of the PLIN gene. Moreover, compared with the previous studies (Zhang et al., 2015; Zhou et al., 2014), the distribution of SNPs of PLIN gene in chicken breeds is evidently different.

\subsection{Relationship of the PLIN gene with some economic traits}

The PLIN gene has been shown to be crucial for some economic traits of animals. Gol et al. (2015) detected a mutation (g.173G $>$ A) of intron 2 in Duroc pigs, which showed some evidence of negative additive and dominant effects on $\mathrm{BW}$ at 120 and 180 days. In sheep, three genetic mutations with major effects on fat-tail weight and tail width have been identified in PLIN (Gao et al., 2012). Fan et al. (2010) found one SNP in the PLIN gene associated with a significantly greater slaughter weight, carcass weight, back fat thickness, and loin muscle area of the $\mathrm{AB}$ genotype, as compared with the $\mathrm{AA}$ and BB genotypes in Qinchuan cattle $(P<0.05)$. In chickens, Zhou et al. (2014) detected three SNPs of PLIN gene and found that the CTA TCA diplotype had a significant effect on living body weight and breast intramuscular fat content of chickens, while the TCA TCA diplotype produced the lowest carcass weight and fatness traits. These findings showed that the PLIN gene had significant effects on carcass and fat traits, similar to its function in different animals. However, research on PLIN gene polymorphisms in chickens has been more focused on slaughter traits and fat deposition, rather than BW, at different ages. Hence, the genetic polymorphisms in exon 5 and partial intron 5 of PLIN gene in Jinmao Hua chickens were screened to discern potential associations between the nucleotide polymorphisms and BW traits.

The findings from the least squares means of different genotypes and diplotypes were interesting. The BW of chickens with the JJ genotype was the heaviest among all the genotypes at each week. Meanwhile, chickens with the CD genotype had the highest mean BW with the exception at week 2. On the whole, chickens with the JJ and CD genotypes performed best in this group. To gain further insight, 322 chickens were divided into eight haplotypes groups using the five SNPs. The haplotypes were found to be significantly associated with BW. Notably, the combination between different haplotypes increased the BW of chickens and the $\mathrm{H} 2 \mathrm{H} 2$ 
Table 6. Correlation analysis between different diplotypes and body weight traits (means $\pm \mathrm{SD}$ ).

\begin{tabular}{lrrrrrr}
\hline Traits & BW0 & BW2 & BW4 & BW6 & BW8 & BW10 \\
\hline$P$ value & 0.093 & 0.430 & 0.067 & $0.027^{*}$ & $0.013^{*}$ & 0.238 \\
\hline H1H1 (152) & $25.02 \pm 2.00$ & $108.72 \pm 16.83$ & $230.75 \pm 40.30$ & $397.37 \pm 65.82$ & $634.82 \pm 86.18$ & $844.96 \pm 113.09$ \\
H1H3 (50) & $25.34 \pm 2.13$ & $106.71 \pm 16.58$ & $228.94 \pm 37.13$ & $407.42 \pm 58.08$ & $632.19 \pm 90.12$ & $851.07 \pm 113.26$ \\
H3H3 (7) & $24.22 \pm 1.29^{2}$ & $96.71 \pm 9.16^{2}$ & $202.31 \pm 45.05^{2}$ & $363.03 \pm 58.09^{2}$ & $574.29 \pm 74.38^{2}$ & $801.20 \pm 71.22^{2}$ \\
H1H4 (22) & $24.41 \pm 2.44$ & $110.77 \pm 16.00$ & $228.17 \pm 34.77$ & $394.31 \pm 56.48$ & $603.62 \pm 93.07$ & $821.07 \pm 107.89$ \\
H3H6 (9) & $25.47 \pm 1.16$ & $112.37 \pm 17.39$ & $223.53 \pm 46.79$ & $390.88 \pm 78.25$ & $618.08 \pm 119.25$ & $813.56 \pm 112.60$ \\
H4H4 (6) & $24.77 \pm 2.69$ & $105.68 \pm 18.04$ & $226.17 \pm 49.21$ & $405.41 \pm 83.61$ & $625.43 \pm 114.26$ & $836.22 \pm 123.81$ \\
H2H2 (33) & $25.87 \pm 2.51^{1}$ & $112.54 \pm 17.47^{1}$ & $238.80 \pm 38.95^{1}$ & $423.64 \pm 58.64^{1}$ & $661.51 \pm 83.08^{1}$ & $869.97 \pm 96.61^{1}$ \\
H2H7 (8) & $24.45 \pm 1.06$ & $109.96 \pm 17.33$ & $217.80 \pm 39.33$ & $379.75 \pm 42.66$ & $606.60 \pm 74.89$ & $834.75 \pm 103.69$ \\
H2H5 (26) & $24.44 \pm 2.07$ & $106.77 \pm 20.66$ & $212.12 \pm 37.70$ & $370.32 \pm 56.65$ & $592.35 \pm 83.86$ & $813.10 \pm 94.25$ \\
\hline
\end{tabular}

Note: ${ }^{1}$ or ${ }^{2}$ italic values are the highest or lowest least squares means, respectively; ${ }^{*} P \leq 0.05$

diplotype was found to be the best combination. For instance, the BW of 1-day-old $\mathrm{H} 2 \mathrm{H} 2$ chickens was $25.87 \mathrm{~g}$, which was $0.27 \mathrm{~g}$ greater than chicks at the same age with the JJ genotype. The BW6 of chickens with the $\mathrm{H} 2 \mathrm{H} 2$ diplotype was 423.64 , which was 8.57 and $16.37 \mathrm{~g}$ greater than JJ and CD genotype, respectively. At BW8, the BW of the $\mathrm{H} 2 \mathrm{H} 2$ diplotype was $661.51 \mathrm{~g}$, which was 7.68 and $19.94 \mathrm{~g}$ greater than chickens with the JJ and CD genotypes, respectively. So, the $\mathrm{H} 2 \mathrm{H} 2$ diplotype is regarded as the most advantageous for chicken BW traits. In addition, the combination of haplotypes also reduced the BW. The $\mathrm{H} 3 \mathrm{H} 3$ chickens had the lowest BW in comparison with other diplotypes, even among all genotypes. As a result, the $\mathrm{H} 3 \mathrm{H} 3$ diplotype was regarded as detrimental to chicken BW traits, suggesting that it should be deleted during cultivation. Haplotype or haplotype block analysis provide a practical solution to resolve the innate problems of the single-marker analysis, such as noisy, unsatisfied, and obscured important localization information (Daly et al., 2001). Both haplotype diversity and the method of SNP selection based on maximum haplotype diversity are always preferred (Huang et al., 2003). According to the data analysis, the SNPs examined in this study may be functionally linked with BW of chickens.

\section{Conclusions}

In conclusion, five SNPs of the PLIN gene were identified in Jinmao Hua chickens that may have an impact on gene function and related with BW traits. Since the present study just demonstrated the effect of partial region of the chicken PLIN gene on body weight, the results of the present study were preliminary. Therefore, it is necessary to determine whether any SNPs exist in other region of the PLIN gene. Meanwhile, the analysis of association between PLIN gene and a wider range performance is also required.

Data availability. The datasets used and/or analyzed during the current study are available from the corresponding author on reasonable request.
Author contributions. TL and JL designed experiments; TL, JL and LD carried out experiments; TL analyzed experimental results; QX analyzed sequencing data and developed analysis tools; TL and JL wrote the manuscript; JW assisted with all.

Competing interests. The authors declare that they have no conflict of interest.

Acknowledgements. This work was supported by National Broiler Industrial and Technology System (no. nycytx-42-G1-05), the Priority Academic Program Development of Jiangsu Higher Education Institutions, China, and the Animal Genetic Breeding and Molecular Design Key Laboratory of Jiangsu Province, China.

Edited by: Steffen Maak

Reviewed by: two anonymous referees

\section{References}

Bickel, P. E., Tansey, J. T., and Welte, M. A.: Pat proteins, an ancient family of lipid proteins that regulate cellular lipid store, Biochim. Biophys. Acta, 1791, 419-440, 2009.

Daly, M. J., Rioux, J. D., and Schaffner, S. F.: High-resolution haplotype structure in the human genome, Nat. Genet., 29, 229-232, 2001.

Fan, H. J., Liu, X. L., and Wang, J.: Analysis of PLIN gene PCRRFLP polymorphism and its association with carcass and adipose traits in meat ducks, J. Acta Agriculturae Boreali-occidentalis Sinica, 20, 12-17, 2011.

Fan, Y. Y., Zan, L. S., and Wang, H. B.: Study on the relationship between polymorphism of PLIN gene and carcass and meat quality traits in Qinchuan cattle, CN J. Anim. and Vet. Sci., 41, 268-273, 2010.

Gao, Z. Y., Lin, P. P., and Yuan, Y. N.: Study on the polymorphism of PLIN gene and its association with tail and slaughter traits in sheep, J. Shanxi Agric. Univ. (Natural Science Edition), 32, 158164, 2012.

Gandolfi, G., Mazzoni, M., and Zambonelli, P.: Perilipin 1 and perilipin 2 protein localization and gene expression study in skeletal 
muscles of european cross-breed pigs with different intramuscular fat contents, Meat Sci., 88, 631-637, 2011.

Gol, S., Ros, F. R., and Zambonelli, P.: Relationship between perilipin genes polymorphisms and growth, carcass and meat quality traits in pigs, J. Anim. Breed. Genet., 133, 24-30, 2015.

Huang, Q., Fu, Y. X., and Boerwinkle, E.: Comparison of strategies for selecting single nucleotide polymorphisms for case/control association studies, Hum. Genet., 113, 253-257, 2003.

Lei, Q. X., Zhou, Y., and Li, J. M.: Association on polymorphisms of intron 5 in PLIN gene with carcass and fat traits in chicken, Chin Anim. Husb. Vet. Med., 38, 142-144, 2011.

Liu, M. F. and Xu, G. H.: Function of PAT family proteins in the lipid metabolism, Prog. Physiol. Sci., 37, 103-107, 2006.

Londos, C., Brasaemle, D. L., and Schultz, C. J.: On the control of lipolysis in adipocytes, Ann. NY Acad. Sci., 892, 155-168, 1999.

Londos, C., Sztalryd, C., and Tansey, J. T.: Role of PAT proteins in lipid metabolism, Biochimie, 87, 45-49, https://doi.org/10.1016/j.biochi.2004.12.010, 2005.

Lü, Y. R., Liu, Y., and Huang, X. R.: Effect of interaction between PLIN gene polymorphisms and open lifestyle intervention on weight-loss in Chinese Han adults, Acta Academiae Medicinae Sinicae, 37, 681-692, 2015.
Vykoukalová, Z., Knoll, A., and Čepica, S.: Porcine perilipin (PLIN) gene: structure, polymorphism and association study in Large White pigs, Czech. J. Anim. Sci., 54, 359-364, 2009.

Wolins, N. E., Quaynor, B. K., and Skinner, J. R.: S3-12, adipophilin, and TIP47 package lipid in adipocytes, J. Biol. Chem., 280, 19146-19155, 2005.

$\mathrm{Xu}, \mathrm{C} ., \mathrm{He}$, J. H., and Xu, G. H.: Perilipin associated with lipid droplets regulates lipolysis, Prog. Physiol. Sci., 37, 221-224, 2006.

Xu, G. H.: Lipolysis molecules control: perilipin phosphorylation initiates lipolysis, CN. J. Pathophysiol., 20, 2380-2381, 2004.

Zhang, H. L., Fan, H. J., and Liu, X. L.: Molecular cloning of the perilipin gene and its association with carcass and fat traits in Chinese ducks, Genet. Mol. Res., 12, 1582-1592, 2013.

Zhang, L., Zhu, Q., and Liu, Y. P.: Polymorphisms in the perilipin gene may affect carcass traits of Chinese meat-type chickens, Asian Australas, J. Anim. Sci., 28, 763-770, 2015.

Zhou, Y., Lei, Q. X., and Han, H. X.: Association on single nucleotide polymorphism of perilipin gene $(P L I N)$ with carcass and fatness traits in luqin chicken, J. Agr. Biot., 22, 1001-1008, 2014. 